\title{
Acknowledgement of Reviewers 2019
}

\section{George Tsakiris ${ }^{1}$}

Published online: 15 February 2020

(C) Springer Nature B.V. 2020

Please join us in thanking all those scientists and experts in the various fields represented in Water Resources Management for devoting time and effort to review the papers that we have been sending them. The Editor-in-Chief, the editorial board and publisher acknowledge the colleagues listed below for their excellent reviews of papers for which final decisions have been made during the period 1 January 2019 to 31 December 2019.

Vasan A

Aminuddin Ab Ghani

Hassan Abbas

Ali Abbasi

Mohammadsadegh Abbasian

Qasem Abdelal

Fahmy Abdelhaleem

Ahmed Abdelkader

Fadi Abdelradi

Ali Abdullah

Rajesh Abhay

Luqman Abidoye

Edo Abraham

Marykutty Abraham

Moema Acselrad

Adebayo Adeloye

Ekundayo Adepehin

Sajal Adhikary

Mohd Shalahuddin Adnan

Haitham Afan

Okechukwu Agbasi

Abdellatif Ahbari
Sainath Aher
Asmadi Ahmad
Ishtiyaq Ahmad
Mohammad Ahmadi
Saima Ahmad
Sakinat Ahmad
Reza Ahmadian
Iman Ahmadianfar
Mahmoud Ahmadpour
Ali Ahmed
Xueshan Ai
Zhipin Ai
Huo Aidi
Hüseyin Akay
Gholamhossein Akbari
Saeid Akbarifard
Fazlullah Akhtar
Mohammad Khaled Akhtar
N.M. Alam
Angelos Alamanos

Shams Al-Amin

Yousif Al-Aqeeli

Seyedhassan Alavinia

Saad Alayyash

Daniel Albiero

Mustafa Al-Furaiji

Ivan Alhama Manteca

Oday Al-Heetimi

Akhtar Ali

Imran Ali

Muhammad Ali

Aidi Alias

Farhad Alizadeh

Hosein Alizadeh

Mohamad Javad Alizadeh

Ahmed Mohammed Sami

Al-Janabi

Loai Aljerf

Ahmed Aljuaidi

Jaber Alkasseh

Mahmoud Al-Khafaji

George Tsakiris

gtsakir@central.ntua.gr

1 Lab. of Reclamation Works and Water Resources Management, National Technical University of Athens, Greece 


\begin{tabular}{|c|c|c|}
\hline Cesar Almeida & Bahram Bakhtiari & John Bredehoeft \\
\hline Faten Al-Najar & Ngandu Balekelayi & Petre Bretcan \\
\hline Basim Al-Obaidy & Hossein Banejad & Rafal Brodziak \\
\hline Nabeel Al-Saati & Polash Banerjee & Christopher Brown \\
\hline Mohanned Al-Sheriadeh & Marcelle Baptista & Philip Brunner \\
\hline Daniel Althoff & Reza Barati & Bruno Brunone \\
\hline Daniel Ames & Alina Barbulescu & Halil Ibrahim Burgan \\
\hline Zahra Amini & Emanuele Barca & Naci Büyükkaracığan \\
\hline Babak Amirataee & Miguel Barrios & Jialiang Cai \\
\hline Maoui Ammar & Kenia Barros & Yi Cai \\
\hline Jafar Amnyattalab & Eyyup Basakin & Ana Paula Camelo \\
\hline Panagiotis Angelidis & Muhammad Basharat & Jose Ruben Campos-Gaytan \\
\hline Mohammad Arab Amiri & Seyyed Nasser Bashi-Azghadi & Shengle Cao \\
\hline Vicky Ariyanti & Hamid Bashiri Atrabi & Charles Carneiro \\
\hline Asaad Armanuos & George Bathrellos & María Carmen Carnero \\
\hline Arfan Arshad & Joost Beckers & Kara Carr \\
\hline Kimberly Artita & Neslihan Beden & Adrian Cashman \\
\hline Mohsen Asadi & Rossano Belladona & Giulio Castelli \\
\hline Antonia Asencio & Vasilis Bellos & Tulin Cetin \\
\hline Seyed Mohammad Ashrafi & Jamel Ben Nasr & Cem Cetinkaya \\
\hline Afshin Ashrafzadeh & M. Benedini & Cristian Chadwick \\
\hline Hasnat Aslam & Hassan Benfetta & Jacob Chandapillai \\
\hline John Asprilla & Bernardino Benito & Winai Chaowiwat \\
\hline Denie Augustijn & Amit Bera & K.W. Chau \\
\hline Ayman Awadallah & Saulo Bezerra & Kwok-Wing Chau \\
\hline Muheeb Awawdeh & Vasanthakumar Bhat & Devatha Chella \\
\hline Taymoor Awchi & Anuran Bhattacharjee & Purushothamman \\
\hline Zeroual Ayoub & Biswa Bhattacharya & Duan Chen \\
\hline Francisco Ayuga & Rabin Bhattarai & Guoqiang Chen \\
\hline Armin Azad & Chandrashekhar Bhuiyan & Jian Chen \\
\hline Muhammad Azam & Seyed Mostafa Biazar & Jiang Chen \\
\hline Hazi Azamathulla & Sumanta Bid & Pan Chen \\
\hline Ali Azareh & Ayla Bilgin & Ying-Jung Chen \\
\hline Arash Azari & Omer Bilhan & Ambe Emmanuel Cheo \\
\hline Ali Azarnivand & $\mathrm{Hu}$ Bin & Alvin Chew \\
\hline Mojtaba Azizi & Deepak Bisht & Kong Chhuon \\
\hline Monte Azmi & Robin Biswas & Po-Kuan Chiang \\
\hline Venkatesh B & Bosko Blagojevic & Madhusudana Rao \\
\hline Ehsan Babanezhad & Claudio Blanco & Chintalacheruvu \\
\hline Meisam Badfar & Ebenezer Boakye & Jung Hyun Choi \\
\hline Lina Bagdziunaite- & Rewa Bochare & Woonsup Choi \\
\hline Litvinaitiene & Janos Bogardi & Chien-Ming Chou \\
\hline Ramin Bahmani & Charles Bong & Roman Cieśliński \\
\hline Tao Bai & Abdelkader Bouderbala & Emin Ciftci \\
\hline
\end{tabular}




\begin{tabular}{|c|c|c|}
\hline Mesut Cimen & Manuel Andres Diaz Loaiza & Carlos Fonseca \\
\hline Gustavo Coelho & Victor Diniz & Yicheng Fu \\
\hline Marcelo Coelho & António Diogo & Mohamad Ali Fulazzaky \\
\hline Theodora Cohen Liechti & Thomas Dippong & Ivan Gabriel-Martin \\
\hline Antonio-Juan Collados-Lara & Selim Dogan & Hadi Galavi \\
\hline Peter Cookey & Funda Dökmen & Armando Gallo Yahn Filho \\
\hline Juan Ignacio Corcoles & Pengfei Du & Guojuan Gan \\
\hline Mirella Costa & Yiheng Du & Liang Gao \\
\hline Huijuan Cui & Anja Du Plessis & Tao Gao \\
\hline Xuefeng Cui & H.F. Duan & Xiang Gao \\
\hline Maria Conceição Cunha & Weili Duan & Francesco Garbo \\
\hline Thibaut Cuvelier & Raphaele Ducrot & Silvestre García De Jalón \\
\hline Mônica Da Hora & Sanjay Dutta & Sudip Gautam \\
\hline Jolanta Dabrowska & Zahir Duz & Savia Gavazza \\
\hline Chao Dai & Naser Ebadati & Venkata Gedela \\
\hline Zhijun Dai & Elham Ebrahimi & Jany George \\
\hline Huseyin Dalkilic & Davidson Egirani & Nyakno George \\
\hline Farhang Daneshmand & Mohammad Ehteram & Bruna Gerônimo \\
\hline Andrea D'Aniello & Alpaslan Ekdal & Mona Ghafouri-Azar \\
\hline Nguyen-Khoi Dao & Mohamed Elfetyany & Reza Ghazavi \\
\hline Alireza Dariane & Ali El-Mahfoodh & Abderrahmane Ghezal \\
\hline Monidipa Das & Ahmed El-Shafie & Mohammad Ali Ghorbani \\
\hline Fernando Das Gracas Braga & Mohamed Eltarabily & Hossein Ghorbanizadeh- \\
\hline Da Silva & Farzad Emami & Kharazi \\
\hline Sonam Dash & Somayeh Emami & Dillip Ghose \\
\hline Josef Datel & Marina Erechtchoukova & Dickson Gidion \\
\hline Evan Davies & Venkata Rathnam Erva & Georgios Gikas \\
\hline José De Araújo & Morteza Esfandyari & Margarita Gil Samaniego \\
\hline J.J. De Felipe & Masoud Eshghizadeh & Ramos \\
\hline Mauro De Marchis & Alfonso Exposito & Carlo Giudicianni \\
\hline Francesco De Paola & Rolando Fabris & Patricia Gober \\
\hline Leydiana De Sousa Pereira & Rasha Fadhil & Marcos Henrique Godoi \\
\hline Surajit Deb Barma & Muhammad Abrar Faiz & Ferhat Gökbulak \\
\hline Jatan Debnath & Yashar Falamarzi & Saeed Golian \\
\hline Hadi Dehghan & Fernando Fan & Ricardo Gomes \\
\hline Roberto Del Teso March & Liangxin Fan & Serdar Göncü \\
\hline Hugo Delottier & Yubing Fan & Jinzhe Gong \\
\hline Girum Demeke & Shiqi Fang & Yicheng Gong \\
\hline Kamalini Devi & Wang Fei & Rebeca González-Villela \\
\hline Gordana Devic & Francisco Fernández & Mustafa Goodarzi \\
\hline Selen Deviren Saygin & Angel Ferrarini & J. Götzinger \\
\hline Ashraf M. Dewan & Jitka Fialová & Venkatesan Govindan \\
\hline Arun Dhawale & Elham Firouznia & Manish Kumar Goyal \\
\hline Danyang Di & Adriana Flores & Francesco Granata \\
\hline
\end{tabular}




\begin{tabular}{|c|c|c|}
\hline Jinjin $\mathrm{Gu}$ & Ardalan Izadi & Hassan Khurshid \\
\hline Mokhtar Guizani & Azizallah Izady & Anthony Kiem \\
\hline Ali Gul & Ashfaque Jafari & Daeha Kim \\
\hline Mustafa Güler & Rahul Jaiswal & Hyun Woo Kim \\
\hline Mert Gunacti & Milad Jajarmizadeh & Sungwon Kim \\
\hline Shenglian Guo & Rehan Jamil & Nagesh Kolagani \\
\hline Aditya Gupta & Jiun-Huei Jang & Venkatesh Kolluru \\
\hline Sushindra Gupta & Saman Javadi & Ekasit Kositsakulchai \\
\hline Vivek Gupta & Tehseen Javed & Cihangir Koycegiz \\
\hline Sunil Gurrapu & Parvathi Jayasankar & Nanabhau Kudnar \\
\hline Sezgin HacisalihoğLu & Varuni Jayasooriya & Mogaraju Jagadish Kumar \\
\hline Sajjad Haider & Ponselvi Jeevaragagam & Neeraj Kumar \\
\hline Wiktor Halecki & Manish Jha & Pankaj Kumar \\
\hline Damon Hall & Bing-Chen Jhong & Pawan Kumar \\
\hline Moshrik Hamdi & Benyou Jia & Vijendra Kumar \\
\hline N.B. Harmancioglu & Guanghui Jiang & Vikram Kumar \\
\hline David Haro Monteagudo & Peng Jiang & Alban Kuriqi \\
\hline Hasrul Hasan & Zhiqiang Jiang & Daniel Prakash Kushwaha \\
\hline Seied Mehdy Hashemy & Lei Jin & Bharat Kusre \\
\hline Shahdany & Naveen Joseph & Davor Kvočka \\
\hline Khalid Hassaballah & G.M. Shamsul Kabir & Surinaidu Lagudu \\
\hline Waqed Hassan & Koosha Kalhor & Chengguang Lai \\
\hline Miao $\mathrm{He}$ & Noha Kamal & Wei Haur Lam \\
\hline Yanhu He & Kandianawati Kandianawati & Philip Langat \\
\hline Yaoyao He & Taeuk Kang & Fares Laouacheria \\
\hline Salim Heddam & Mitthan Kansal & Claudia Lardizabal \\
\hline Bagher Heidarpour & Kanak Kanti Kar & William Laton \\
\hline Jun-Haeng Heo & Fadi Karam & Agostino Lauria \\
\hline Younggu Her & Omid Karami & Seungho Lee \\
\hline Mahdi Hesami Afshar & Akbar Karimi & Sangho Lee \\
\hline Martin Hipondoka & Keivan Karimlou & Earl Lewis \\
\hline Akbar Hossain & Ahmed Karmaoui & Cuimei Li \\
\hline Farzad Hosseini Hossein & Konstantinos Katsifarakis & Hui Li \\
\hline Abadi & Suparana Katyaini & Jiahong Li \\
\hline Zeinab & R. Kaviyarasan & Junfeng Li \\
\hline Hosseinzadehghazichaki & Nerantzis Kazakis & Peiyue Li \\
\hline Shengzhi Huang & Nerantzis Kazakis & Peng Li \\
\hline Xiaorong Huang & Hamed Ketabchi & $\mathrm{Xia} \mathrm{Li}$ \\
\hline Yenchen Huang & Mohd Yawar Khan & Xungui Li \\
\hline Ijaz Hussain & Suresh Khandelwal & Yongping Li \\
\hline Ali Reza Ildoromi & Omar Khashman & Gustavo Lima \\
\hline Aleksandra Ilić & Puneet Khatavkar & Hebin Lin \\
\hline Md Monirul Islam & Rajesh Kherde & Kairong Lin \\
\hline Zahidul Islam & Mojtaba Khoshravesh & Tonino Liserra \\
\hline
\end{tabular}




\begin{tabular}{|c|c|c|}
\hline Bojun Liu & Gagan Matta & Paula Niinikoski-Fußwinkel \\
\hline Dedi Liu & Jonatas Mattos & Alireza Nikbakht Shahbazi \\
\hline Haixing Liu & Joana Medeiros & Mohammad Reza Nikoo \\
\hline Jiahong Liu & Nazli Mehzad & Olga Nitcheva \\
\hline Qiang Liu & Yadong Mei & Felix Njagi \\
\hline Xingcai Liu & Muhammad Sohail Memon & Xizhi Nong \\
\hline Yong Liu & Yaobin Meng & Denis Nono \\
\hline Yuyu Liu & Jailakshmi Menon & Roohollah Noori \\
\hline Carmen Lizarraga & Daniel Merchán & M.Masud Noruzi \\
\hline Abbas Ljam & Shein Mezour & Tiago Novais \\
\hline D.P. Loucks & M.Q. Mirza & Umut Okkan \\
\hline Athanasios Loukas & Firdaus Mohamad Hamzah & Marcelo Olivares \\
\hline Huiting Lu & Mahmoud Mohammad & Padam Omar \\
\hline Bin Luo & Rezapour Tabari & Ibrahim Oroud \\
\hline Jungang Luo & Babak Mohammadi & Nazife Oruc Baci \\
\hline Mingjie Luo & Ahmed Mohammed & Faridah Othman \\
\hline Pingping Luo & Sheelabhadra Mohanty & Laila Oualkacha \\
\hline Chinh Luu & Jose-Luis Molina & Hajer Ouni \\
\hline Gustavo Lyra & Majid Montaseri & Ceyhun Özçelik \\
\hline Janga Reddy M. & Amir Mohammad Moradi & Ayfer Özdemir \\
\hline Chuanming Ma & Hamed Moradi & Arda Özen \\
\hline Jaafar Maatooq & Eliana Moraes-Santos & Ismail Özölçer \\
\hline Aline Machado & Mojtaba Moravej & Sreeja P. \\
\hline Sunandana Reddy & Sayed Masood Mostafavi & Sudheer Padikkal \\
\hline Machireddy & Darani & Victor Luis Padilha \\
\hline Viliam Macura & Noryanti Muhammad & Roberta Padulano \\
\hline Mohamad Reza Madadi & Arunangshu Mukherjee & Nirav Pampaniya \\
\hline Darrien Mah & Asmita Murumkar & Pankaj Pandey \\
\hline Mohamed Mahmoud & Christos Myriounis & Dinagara Pandi \\
\hline Maysam Majidi & Dimitrios Myronidis & Chrysaida-Aliki \\
\hline Anurag Malik & Ramsundram N & Papadopoulou \\
\hline Shaikh Abdullah Al Mamun & Somayeh Naderi & Daeryong Park \\
\hline Hossain & Magesh Nagarajan & Behnam Parmas \\
\hline Zahra Mansouri & Mohammad Najafzadeh & Atefeh Parvaresh Rizi \\
\hline Joyce Maphanyane & I. Nalbantis & Sharad Patel \\
\hline Jeffrey Denzil Marak & Bijaya Kumar Nanda & Vihang Pathare \\
\hline Paweł Marcinkowski & Touraj Nasrabadi & Aakash Pawar \\
\hline Alireza Mardookhpour & Carolina Natel De Moura & Ahmad Payab \\
\hline Milica Markovic & Mohammad Nazeri Tahroudi & Flávio Pedro Filho \\
\hline Włodzimierz Marszelewski & Mehdi Nezhad Naderi & Roya Peirovi \\
\hline Carlos Martinez & Keziah Ngugi & Genevieve Pelletier \\
\hline Vinicius Otto Marzall & Xiaokuan Ni & Modesto Pérez-Sánchez \\
\hline Reza Mastouri & Majid Niazkar & Christos Petalas \\
\hline Nametso Matomela & Tewodros Assefa Nigussie & Elnaz Pezeshki \\
\hline
\end{tabular}




Izabela Piegdoń
Katarzyna Pietrucha-Urbanik
Cicero Pimenteira
Jamshid Piri
Concepcion Pla
Ioana Popescu
Maria Manuela Portela
Yavar Pourmohamad
M.N. Prakash
A.D. Prasad
Virendra Proag
Farhad Qaderi
Junyu Qi
Dragan Radivojevic

Sujay Raghavendra N

Dariush Rahimi

Majid Rahimpour

Subash Rai

Pradeep Rajput

Arturo Ramos-Bueno

Suresh Reddy

Daniel Reddythota

Maria Fernanda Reyes Perez

Abolfazl Rezaei

Abdul Razaq Rezaei

Hossien Riahi Madvar

Jose Ribas

Renato Ribeiro

Sultan Rizvi

Amir Roshan

Mousumi Roy

Mehman Rzayev

Mohsen Saadat

Ali Saber

Sina Sadeghfam

Fouad Saeed

Iraj Saeedpanah

Mohammadreza Safaei

Rajeev Sahay

A.Ufuk Sahin

Bibhuti Sahoo

Satiprasad Sahoo

Mohammad Salarian

Abrari Salleh

Saad Sammen
Rodrigo Sánchez-Román

Francisco-Javier Sánchez-

Romero

Daniel Sant'Ana

Vijay Santikari

Claudia Santos

Jay Sarraf

Tabinda Sattar

Andre Schardong

Mahdi Sedighkia

Satheeshkumar Sekar

Arsalan Sepehri

Kashif Shaad

Zia Ul Hasan Shah

Abdol Aziz Shahraki

Isam Shahrour

Rahaf Shakko

Ahmad Sharafati

Mohammad Reza Sharifi

Uttam Sharma

Alemayehu Shawul

Haiyun Shi

David Ching-Fang Shih

Ehsan Shirangi

Pedro Silva

Ajay Singh

Ajit Pratap Singh

Chandra Singh

Prateek Kumar Singh

Pushpendra Singh

Marcus Siqueira Campos

Murugesu Sivapalan

Weiwei Song

Xiaomeng Song

Jaysukh Songara

Mike Spiliotis

K. Srinivasa Raju

Sunil Srivastava

Asep Suheri

Jueyi Sui

Sadeq Oleiwi Sulaiman

Ali H. Ahmed Suliman

Qamar Sultana

Rebeka Sultana

Feifei Sun
Peng Sun

Shanlei Sun

Thanaporn Supriyasilp

Wiesław Szulczewski

Nata Tafesse

Arash Tafteh

Wardah Tahir

Yaogeng Tan

Guolei Tang

Hasan Tatli

Sara Taviani

Georgios Tentes

Meseret Teweldebrihan

Roshni Thendiyath

Dimitris Tigkas

Wai Ming To

Rahul Todmal

Ali Torabi Haghighi

María Nariné Torres Cajiao

Slavisa Trajkovic

Thong Anh Tran

Trinh Tran

Ali Triki

Gaurav Tripathi

Flavio Trojan

Paraskevas Tsangaratos

Stavroula Tsitsifli

Michael Tumbare

Mustafa Erkan Turan

Duygu Ulker

Gokcen Uysal

Babak Vaheddoost

Nariman Valizadeh

Tom Van Der Voorn

Divyesh Varade

Deepa Varghese

Vahid Varshavian

Saeed Vazifehkhah

Valliappan Vedha Narayanan

Pablo Velarde Rueda

Rambha Venkataramana

Anand Verdhen

Carlos Verduzco

Edson Vieira

Gonzalo Villa-Cox 


$\begin{array}{lll}\text { María C. Villarín } & \text { Wei Xu } & \text { Xiang Zeng } \\ \text { Alejandra Volpedo } & \text { Yunqing Xuan } & \text { Yebegaeshet Zerihun } \\ \text { Pawan Wable } & \text { Mehdi Yaltaghian Khiabani } & \text { Amin Zeynolabedin } \\ \text { Fei Wang } & \text { Hexiang Yan } & \text { Xiaoyan Zhai } \\ \text { Hongrui Wang } & \text { Lihu Yang } & \text { Luxin Zhai } \\ \text { Hui Wang } & \text { Liming Yao } & \text { Bing Zhang } \\ \text { Huiliang Wang } & \text { Zaher Yaseen } & \text { Chen Zhang } \\ \text { Juan Wang } & \text { Mohammad Reza Yazdani } & \text { Chuan Cai Zhang } \\ \text { Lei Wang } & \text { Haw Yen } & \text { Ruilian Zhang } \\ \text { Shun-Jiu Wang } & \text { Burhan Yildiz } & \text { Wei Zhang } \\ \text { Wenchuan Wang } & \text { Baris Yilmaz } & \text { Xu Zhao } \\ \text { Yonggui Wang } & \text { Sheng Yu } & \text { Binghui Zheng } \\ \text { Zhaoli Wang } & \text { Tingchao Yu } & \text { Ming Zhong } \\ \text { Huaibin Wei } & \text { Xue Yu } & \text { Jian-Lan Zhou } \\ \text { Fikadu Worku } & \text { Meihua Yuan } & \text { Jianzhong Zhou } \\ \text { Mekete Wosenie } & \text { Wenlin Yuan } & \text { Weibo Zhou } \\ \text { Hui Wu } & \text { Javad Zahiri } & \text { Jianting Zhu } \\ \text { Penghai Wu } & \text { Omid Zamani } & \text { Li Zongjie } \\ \text { Yanqing Wu } & \text { Sarvin Zamanzad Ghavidel } & \text { Mohammad Zounemat- } \\ \text { Huijie Xiao } & \text { Kerstin Zander } & \text { Kermani } \\ \text { Liu Xiuli } & \text { Mohanna Zarei } & \text { Shaher Zyoud } \\ \text { Shengqing Xu } & \text { Demetrio Antonio Zema } & \\ & & \end{array}$

We apologize for any errors or inadvertent omissions. 